\title{
Management of Eye Trauma for the Primary Care Physician
}

\author{
Jonathan Y. Rho, BS, Stephen C. Dryden, MD, Brian M. Jerkins, MD, and \\ Brian T. Fowler, MD
}

Eye trauma is frequently seen by non-ophthalmology providers. This article elucidates a methodological approach to eye trauma. The first step is to address any life-threatening conditions. Then a focused history and exam is discussed, starting externally. Then, key history, physical, pathophysiology, and basic management of common, serious eye injuries are discussed: chemical injury, orbital fractures, open globe, traumatic hyphema, retinal detachment, traumatic optic neuropathy, and eyelid laceration. Finally, we highlight the practicality of telemedicine for areas where ophthalmology coverage is lacking. (J Am Board Fam Med 2021;34:1018-1029.)

Keywords: Chemical Burns, Eye Injuries, Hyphema, Lacerations, Ophthalmology, Optic Nerve Injuries, Orbital Fractures, Retinal Detachment, Soft Tissue Injuries, Telemedicine, Triage

\section{Introduction}

According to 2010 data from the Nationwide Emergency Department Sample, a main ophthalmic diagnosis represented 1,996,735 emergency department (ED) visits (1.5\%) out of a total $128,970,364$ ED visits in the United States. ${ }^{1}$ Although ophthalmic care is common in the ED, there is a lack of ophthalmology coverage in many departments. ${ }^{2,3}$ Often, the initial evaluation is performed by a non-ophthalmologist. Triage is focused on addressing life then vision-threatening conditions. It is important for the triaging provider to perform a basic eye examination and recognize conditions that require prompt consultation from an eye care provider. The goals of this article are to establish a methodological approach to eye trauma for non-eye care providers, identify vision-threatening conditions that require urgent subspecialty care, and initiate proper management of common eye injuries.

This article was externally peer reviewed.

Submitted 23 March 2021; revised 10 May 2021; accepted 11 May 2021.

From the Hamilton Eye Institute, University of Tennessee Health Science Center, Memphis, TN (RYO, SCD, BMJ, BTF).

Funding: None.

Conflict of interest: None.

Corresponding author: Jonathan Rho, BS, Hamilton Eye Institute, University of Tennessee Health Science Center, Memphis, TN 38103 (E-mail: rho.jonathan@gmail.com).

\section{Approach for Triaging Provider}

The approach and utility of some of the physical examination techniques and ancillary testing may depend on the comfort of the triaging provider and setting of practice. As outlined in Figure 1, a stepwise approach can help the triaging provider determine when an urgent ophthalmology consultation is necessary (Figure 1). The assessment begins with addressing life-threatening issues by stabilizing and transferring the patient to appropriate care if the patient has unstable vital signs, altered mental status, or serious non-ocular injuries. ${ }^{4}$ If stable, assess for vision-threatening conditions. If chemical eye injury is suspected, then immediate copious irrigation is imperative. For other injuries, document the following in a systematic fashion: general history, ocular history, external examination, and internal examination. To avoid missing injuries, examine both eyes. ${ }^{5}$ First, document a general history: medical conditions, prior surgeries, drug allergies, current medications, complications with anesthesia, time of last liquid or food, and tetanus immunization status. ${ }^{4,5}$ Then, obtain an ocular history: the mechanism, setting, and timing of injury; the composition of intraocular foreign body (IOFB) if applicable; the use and final condition of eyewear; symptoms; ocular medications; and prior ocular history including preinjury visual acuity (VA) or a change from baseline. ${ }^{4,6}$

Begin the physical examination by testing the uncorrected VA and gross visual field with finger 
Figure 1. Flowchart showing the approach to eye trauma for the triaging provider.

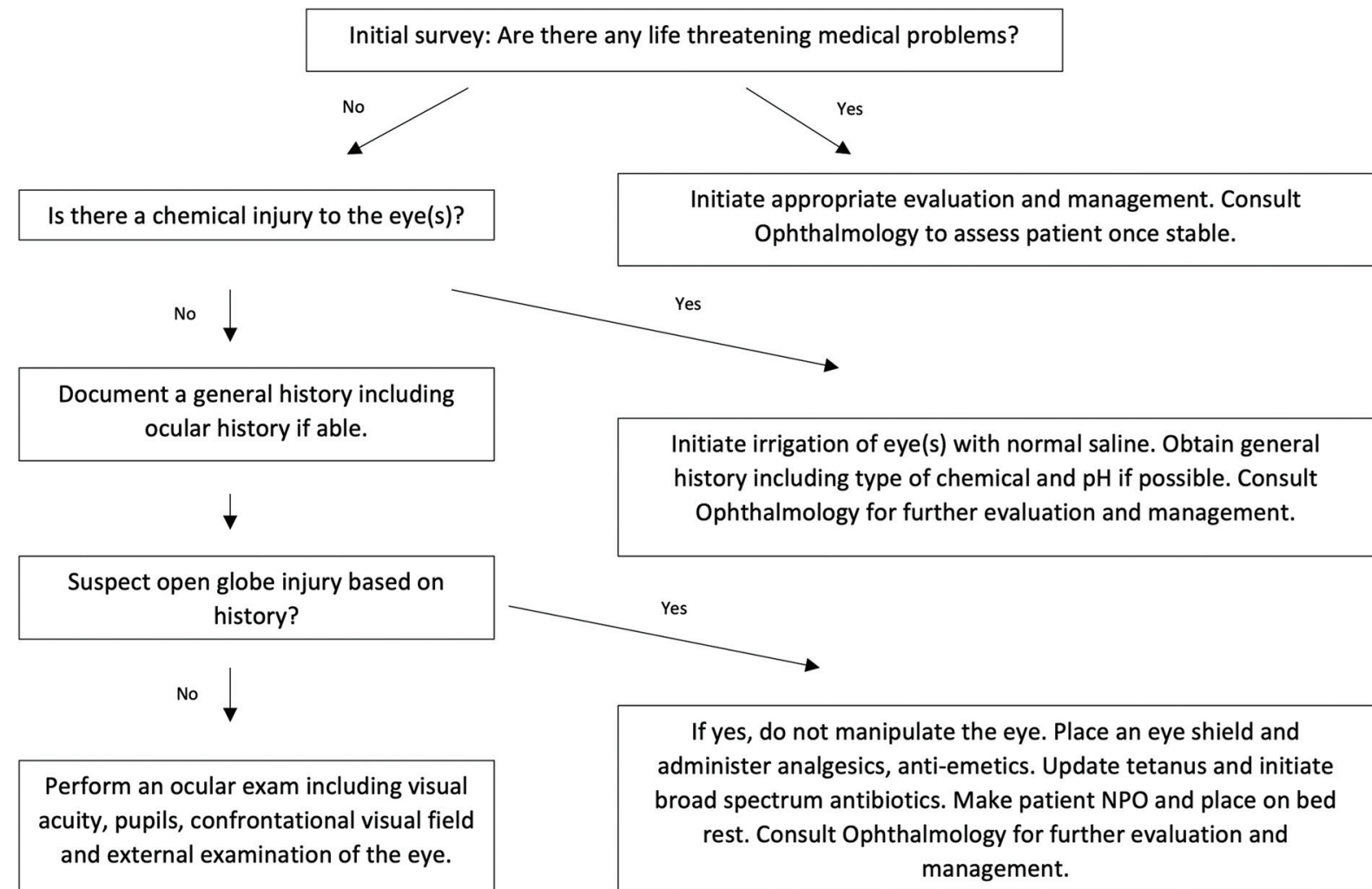

Initiate management and refer to Ophthalmology as an outpatient

Consult Ophthalmology for further evaluation and management. confrontation for each eye. For severe injuries with poor VA, using a semiquantitative scale of counting fingers, hand motion, light perception, and no light perception progressively. ${ }^{7}$ If the VA is worse than expected, the threshold for referral should be lowered. ${ }^{8}$ In the absence of an open globe, intraocular pressure (IOP) should be measured if appropriate equipment is available. Pressures between $11 \mathrm{mmHg}$ and $21 \mathrm{mmHg}$ are considered normal. Mechanical outflow obstruction, hyphema, and orbital compartment syndrome can cause elevated IOP, while low pressures are worrisome for an open globe, ciliary body injury, or retinal detachment. ${ }^{9-11}$ Visual field abnormalities on finger confrontation may indicate optic nerve, retinal injury, or presence of intracranial pathology. ${ }^{5}$

External inspection begins with the head, scalp, periorbital tissue, and eyelids, noting lacerations, ecchymosis, edema, crepitus, dysesthesias, and ptosis. ${ }^{5}$ Note the presence or absence of exophthalmos, enophthalmos, or asymmetry. ${ }^{5}$ Look for blood, swelling, laceration, or exposed tissue in the conjunctiva. ${ }^{5,7}$ Fluorescein staining under cobalt blue light can help identify epithelial defects; leakage of clear aqueous humor mixes with fluorescein dye, diluting the color and creating a stream (Seidel positive) suggestive of a full-thickness injury. ${ }^{5,7}$ 
Figure 2. Alkali burn. External photograph of an eye with alkali burn with chemosis $(*)$, subconjunctival hemorrhage $\left({ }^{\wedge}\right)$, epithelial defect with fluorescein staining (\#), limbal stem cell damage (\&), and pseudohypopion (\$).

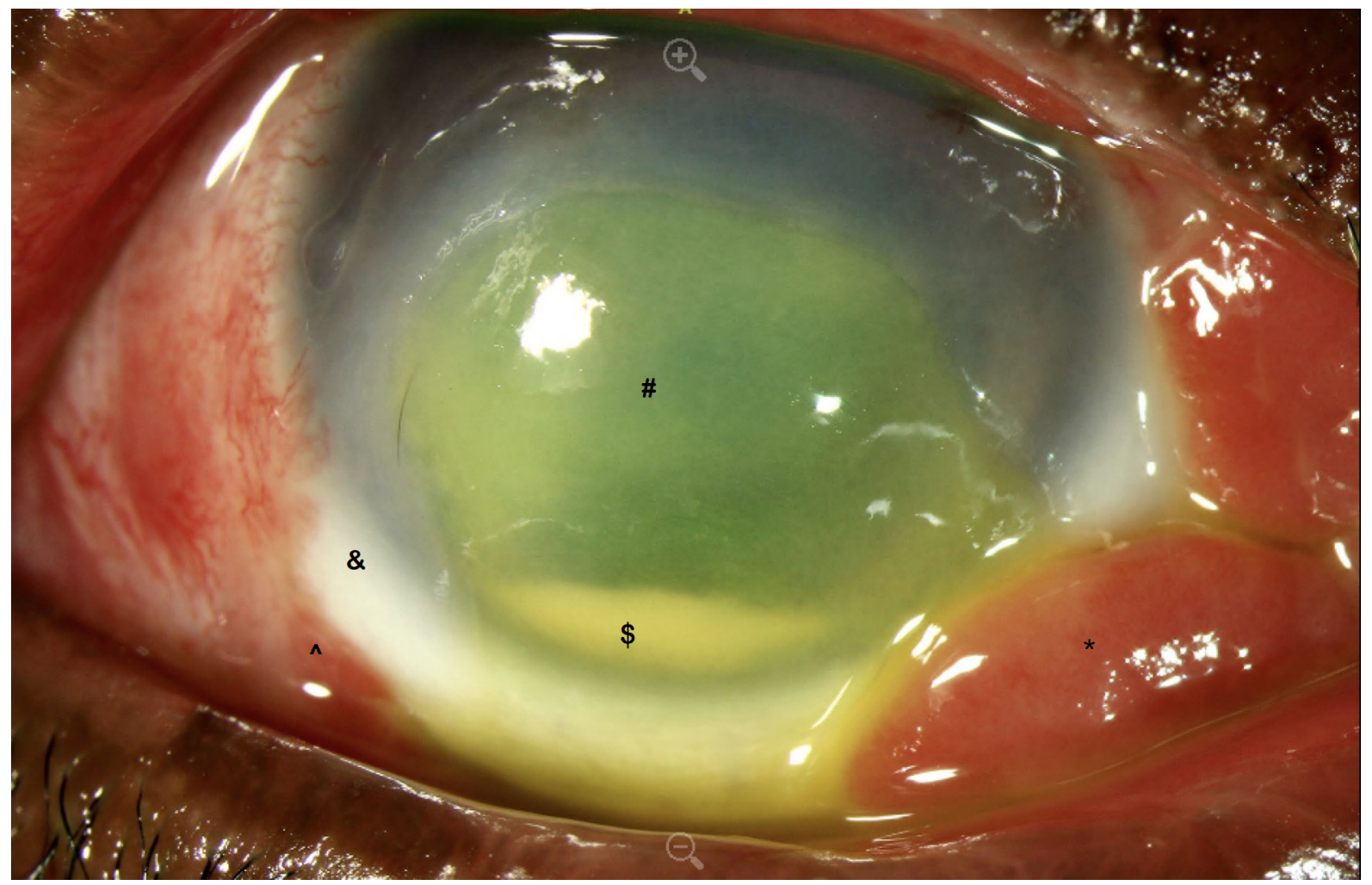

The internal physical examination may be limited based on available equipment, provider comfort, and patient presentation. Assess the anterior chamber for hyphema, inflammation, and depth. Deepening can occur with scleral rupture, iridodialysis, and posterior dislocation of the lens, while shallowing can be seen with vitreous prolapse, corneoscleral wound leak, angle-closure, and anterior dislocation of the lens. ${ }^{4}$ Note the size, shape, and symmetry of the iris and pupil. ${ }^{5,7}$ In a patient with a relative afferent pupillary defect (rAPD), both pupils dilate when the affected eye is exposed to light during the swinging light test and may suggest optic nerve injury or severe retinal detachment. ${ }^{12} \mathrm{~A}$ red object that seems gray (red desaturation) suggests optic nerve damage. ${ }^{4}$

On recognizing a vision-threatening condition, providers should initiate basic management when appropriate and promptly consult an ophthalmologist. Clinicians may follow the systematic approach of obtaining a general history, ocular history, external examination, and internal examination for all conditions except for chemical eye injuries, where copious irrigation should begin immediately.

\section{Ancillary Testing}

In acute trauma, imaging is helpful because many factors, such as periorbital edema, media opacification, and altered mental status may limit clinical evaluation. Although magnetic resonance images (MRIs) provide superior images for soft tissue, they are infrequently used for acute ocular trauma and are contraindicated with metallic IOFBs. ${ }^{13}$ Compared with MRIs, computed tomography (CT) scans are widely available, cheaper, faster, and provide less motion artifact and claustrophobia. ${ }^{5}$ Thin-sliced helical CT scan is the preferred imaging modality for ocular trauma and has antiquated plain radiography due to its higher sensitivity for fractures and soft tissue injuries. ${ }^{13,14} \mathrm{CT}$ scans are the best modality to evaluate orbital fractures, and 3-dimensional CT reconstruction can guide subsequent surgical management. ${ }^{5,14}$ However, CT scans provide inferior images of intraocular structures compared with ultrasound (US). ${ }^{5}$ US provides images without radiation exposure in a time and cost-efficient manner. ${ }^{5}$ Particularly helpful in patients with media opacification, US can detect lens dislocation, IOFB, retrobulbar or vitreous 
Figure 3. Left inferior rectus entrapment. External photography composite of the primary positions of gaze of a patient with left inferior rectus entrapment and lower eyelid ecchymosis demonstrating vertical movement deficits in the left eye. Note, in primary position, the fixating right eye is higher relative to the hypotropic left eye, as appreciated by the uneven corneal light reflex (Hirshberg test).
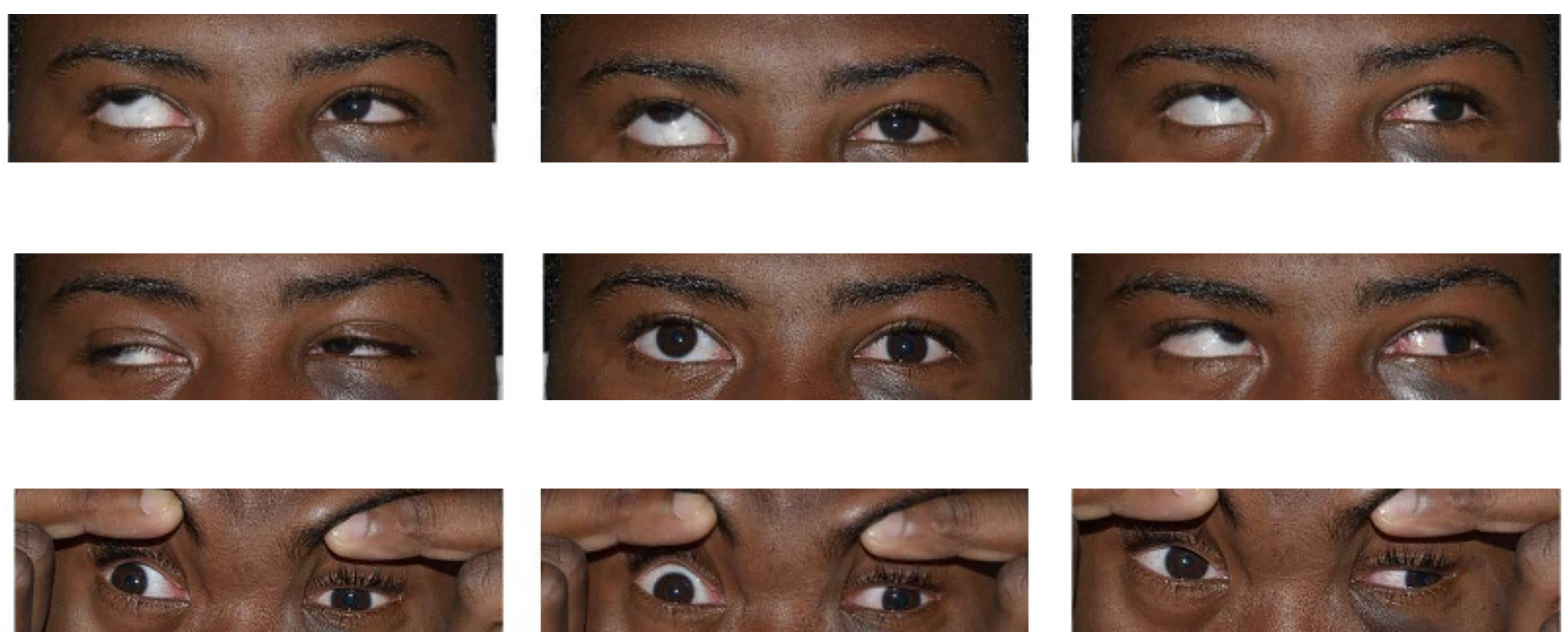

hemorrhage, and retinal detachment. ${ }^{5,7}$ However, because of direct contact with the eyelids, US is contraindicated if an open globe is suspected. ${ }^{5}$

\section{Chemical Injury}

The severity of ocular damage from chemicals depends on the type of agent, volume, and duration of exposure. Alkaline agents saponify fatty acids of the corneal membrane, causing liquefactive necrosis and allowing deep corneal penetration. The damage can reach limbal stem cells and prevent normal healing (Figure 2). On the other hand, coagulative necrosis from denatured proteins relatively protects the eye from deep acid penetration. ${ }^{15,16}$ Because the time between chemical exposure and irrigation has the most influence on prognosis, irrigation with isotonic saline should precede ocular evaluation. ${ }^{17}$ Irrigation should continue until $\mathrm{pH}$ testing at the conjunctival fornix maintains a $\mathrm{pH}$ between 7.0 to 7.4. Then, the eye can be examined. ${ }^{16}$ Topical anesthetics can attenuate pain to optimize patient comfort. ${ }^{17} \mathrm{~A}$ Morgan Lens is a sterile plastic lens connected to tubing to provide continuous irrigation of the eye without constant supervision and effectively washes despite blepharospasms (eyelid spasms). ${ }^{18}$

\section{Orbital Fractures}

Blunt trauma most commonly fractures the orbital floor and medial orbital wall. ${ }^{19}$ The mechanism is explained by both the buckling and hydraulic theory, where force is transmitted through the bone and orbital soft tissues, respectively. ${ }^{20}$ Entrapment of the inferior rectus muscle or adjacent soft tissue can restrict vertical eye movement, cause diplopia, and induce bradycardia due to the oculocardiac reflex. ${ }^{19-22}$ (Figure 3) Resultant edema and orbital hemorrhage may also restrict ocular motility. ${ }^{19-22}$ Damage to the infraorbital nerve may cause altered sensation between the ipsilateral inferior orbital rim to the upper lip. ${ }^{19}$

Entrapment of the medial rectus can restrict horizontal eye movement. ${ }^{21}$ Examination may reveal retraction of the globe and narrowing of the palpebral fissure on attempted abduction (PseudoDuane's Retraction Syndrome). ${ }^{23}$ Medial wall trauma can damage the nasolacrimal drainage system and medial canthal tendon resulting in an overflow of tears onto the face (epiphora) and widened intercanthal distance, respectively. ${ }^{22,24}$

Thin cut CT scan of the orbit is the imaging modality of choice because it can detect involvement with the optic canal, presence of orbital hemorrhage, and visualize entrapment of the rectus muscles. ${ }^{22}$ Patients should avoid blowing their nose for a few weeks to decrease the risk of orbital emphysema. (Figure 4) The use of antibiotics for orbital fractures is controversial with no significant evidence but may prevent orbital cellulitis. ${ }^{22}$ Surgical intervention is indicated for persistent diplopia with evidence of entrapment, enophthalmos $>2 \mathrm{~mm}$, and a fracture that involves more than $50 \%$ of the orbital floor. ${ }^{22}$ Surgical repair is best if performed within 2 weeks of the injury before scarring begins. ${ }^{22,25}$ 
Figure 4. Orbital fracture with emphysema. Coronal CT scan of the orbit demonstrating right orbital floor $(*)$ and medial wall fracture $\left({ }^{\wedge}\right)$ with orbital emphysema (\#).

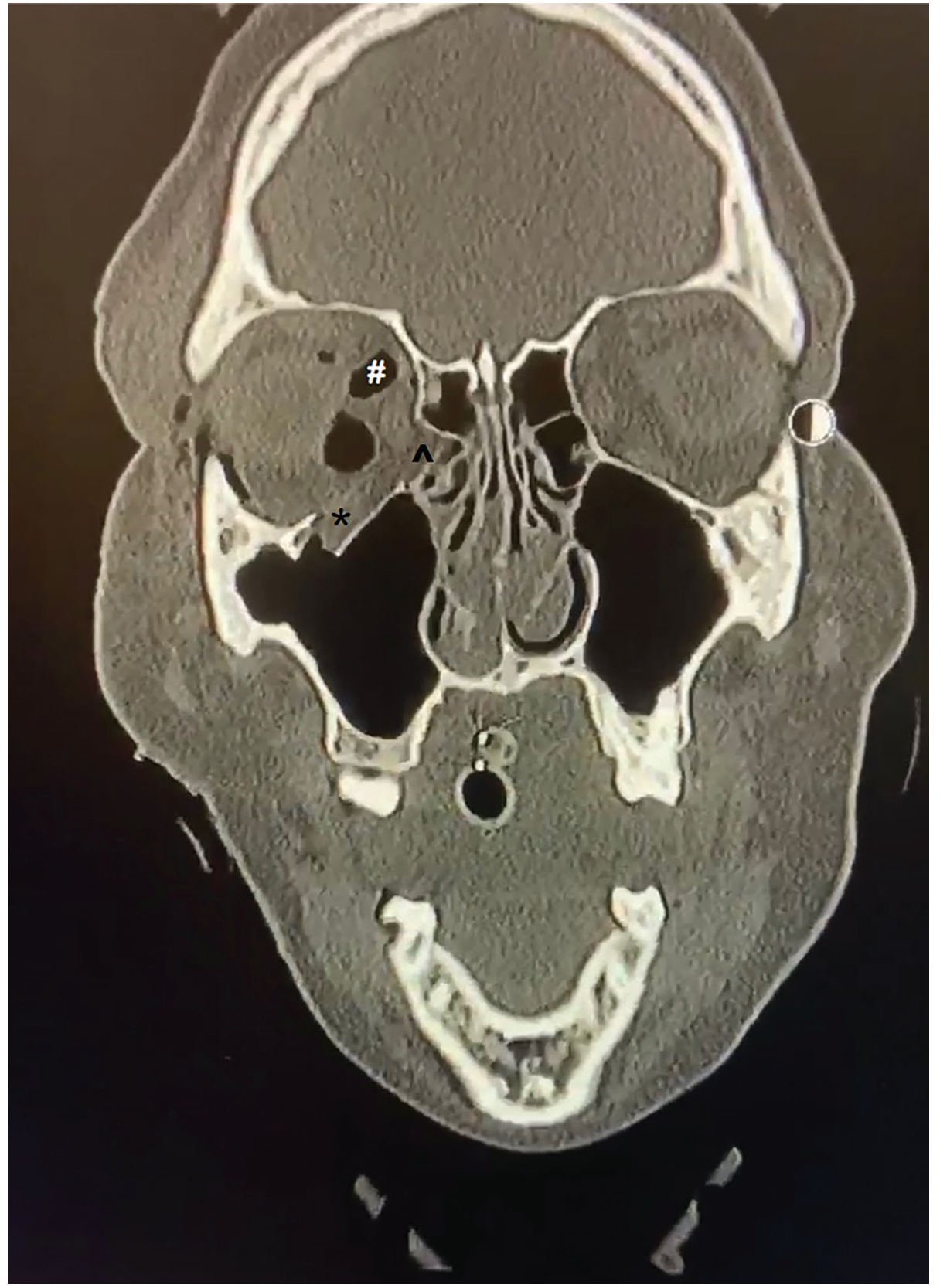

\section{Orbital Compartment Syndrome}

Because the orbit is an enclosed space, a rise in volume from an acute hemorrhage or soft tissue swelling can lead to a rapid increase in intraorbital pressure. Compartment syndrome occurs when intraorbital pressure exceeds arterial perfusion pressure of the optic nerve, ultimately resulting in permanent vision loss within 90 minutes. ${ }^{26}$ This is a clinical diagnosis and should be suspected with a history of trauma, double vision, or proptosis and signs of decreased VA, elevated IOP, rAPD, ophthalmoplegia, periocular edema, and evidence of increased orbital pressure such as tense eyelids and resistance to retropulsion (Figure 5). If orbital compartment syndrome is suspected, ophthalmology should be consulted urgently to evaluate and decompress the orbit if necessary. ${ }^{11}$ 
Figure 5. Orbital compartment syndrome. External photograph of the right eye with orbital compartment syndrome with signs of periorbital edema $(*)$, hemorrhagic chemosis $\left({ }^{\wedge}\right)$, and proptosis.

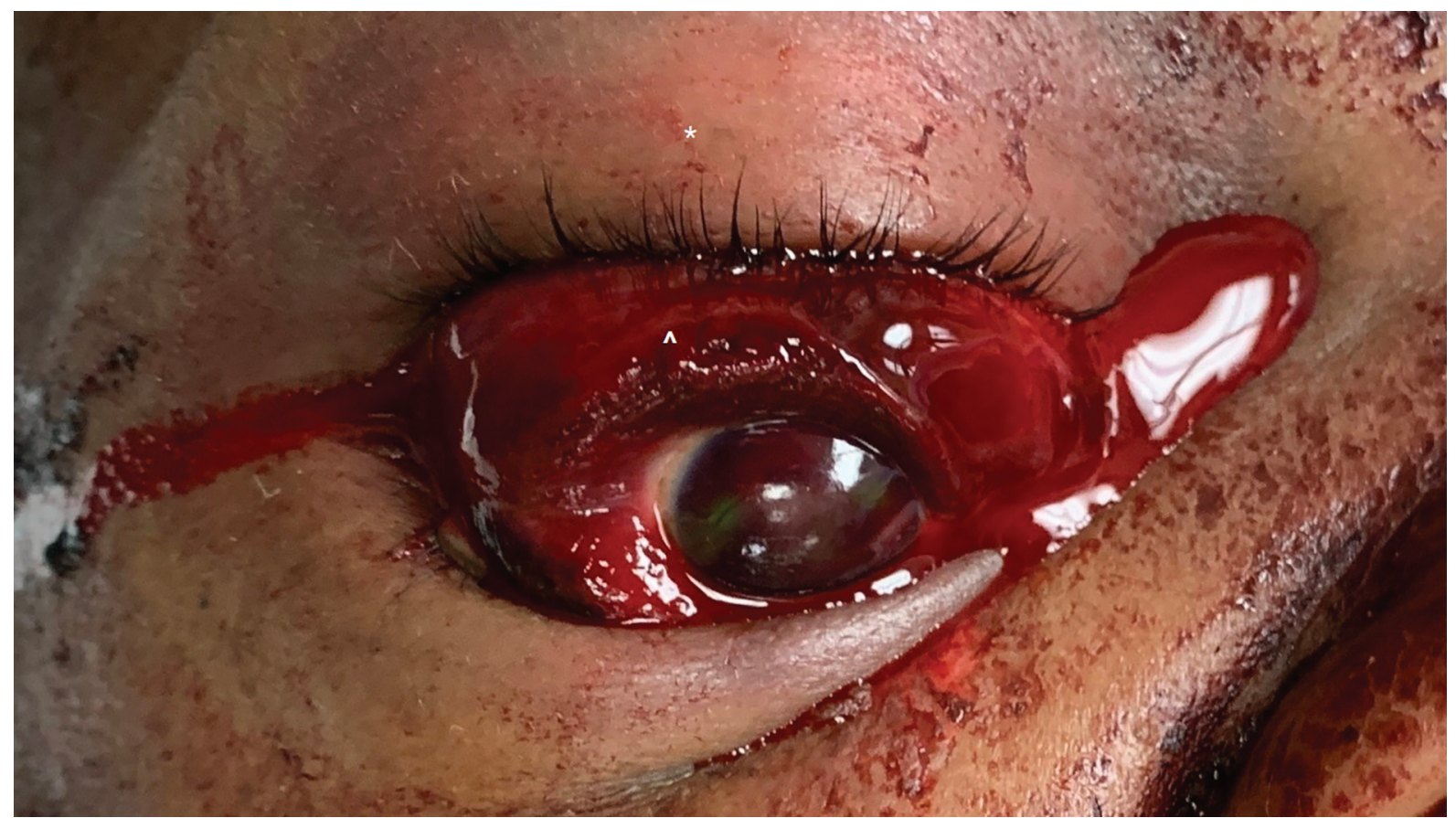

\section{Open Globe Injury}

A full-thickness injury results in an open globe. The patient may present with sudden eye pain and vision loss after trauma. An examination may show decreased visual acuity, irregular-shaped pupil, deepening or shallowing of the anterior chamber, extrusion of vitreous with loss of eyeball contour, and ocular hypotony (Figure 6). ${ }^{4,27}$ To prevent extrusion of intraocular content, maneuvers that increase IOP, such as tonometry and eyelid retraction, should be avoided. Removal of foreign objects should be deferred, and the affected eye protected with an eye shield. The patient should be given antiemetic and pain medication and remain NPO with bed rest elevated to 30 degrees. ${ }^{28}$ The patient's tetanus should be up to date and broad-spectrum antibiotics administered to decrease the risk of endophthalmitis. ${ }^{28}$

\section{Traumatic Hyphema}

Blood in the anterior chamber is known as hyphema. Ocular contusion causes anteroposterior compression of the globe with simultaneous equatorial elongation. This expansion can damage the iris or ciliary body vessels with subsequent bleeding. ${ }^{29,30}$ Hyphemas range from microscopic to involving the whole anterior chamber and are graded from $0-\mathrm{IV}$, respectively (Figure 7 and Figure 8). Complications include obstruction of trabecular meshwork with increased IOP, optic atrophy, rebleeding, synechiae, and corneal blood staining. ${ }^{30}$

Initial management includes elevating the head of the bed to 30 degrees, placement of an eye shield, and bed rest. Avoid non-steroidal anti-inflammatory drugs (NSAIDs) or aspirin products because their platelet inhibiting properties increase bleeding. Nausea and vomiting should be controlled to avoid increases in IOP. In the absence of narrow-angle glaucoma, a mydriatic can attenuate photophobia. Topical prednisolone acetate $1 \%$, in addition to topical long-acting cycloplegics, such as atropine, can decrease IOP, synechiae development, and risk of secondary bleeding. ${ }^{30}$ The risks and benefits of discontinuing anticoagulation should be discussed with an internist. Sickle cell screening should be considered in African American patients or other suspect individuals. ${ }^{30}$ In patients with sickle cell disease or trait, avoid medications that promote sickling, such as acetazolamide, because sickled RBCs trapped in the trabecular meshwork raises IOP. ${ }^{8}$ Inpatient management is recommended for hyphema with grade III or IV, bleeding coagulopathy, or sickle cell comorbidity. Patient adherence to activity limitation, medications, and ophthalmology follow- 
Figure 6. Corneal laceration. External photograph of the left eye with a full-thickness corneal laceration with uveal prolapse $(*)$.

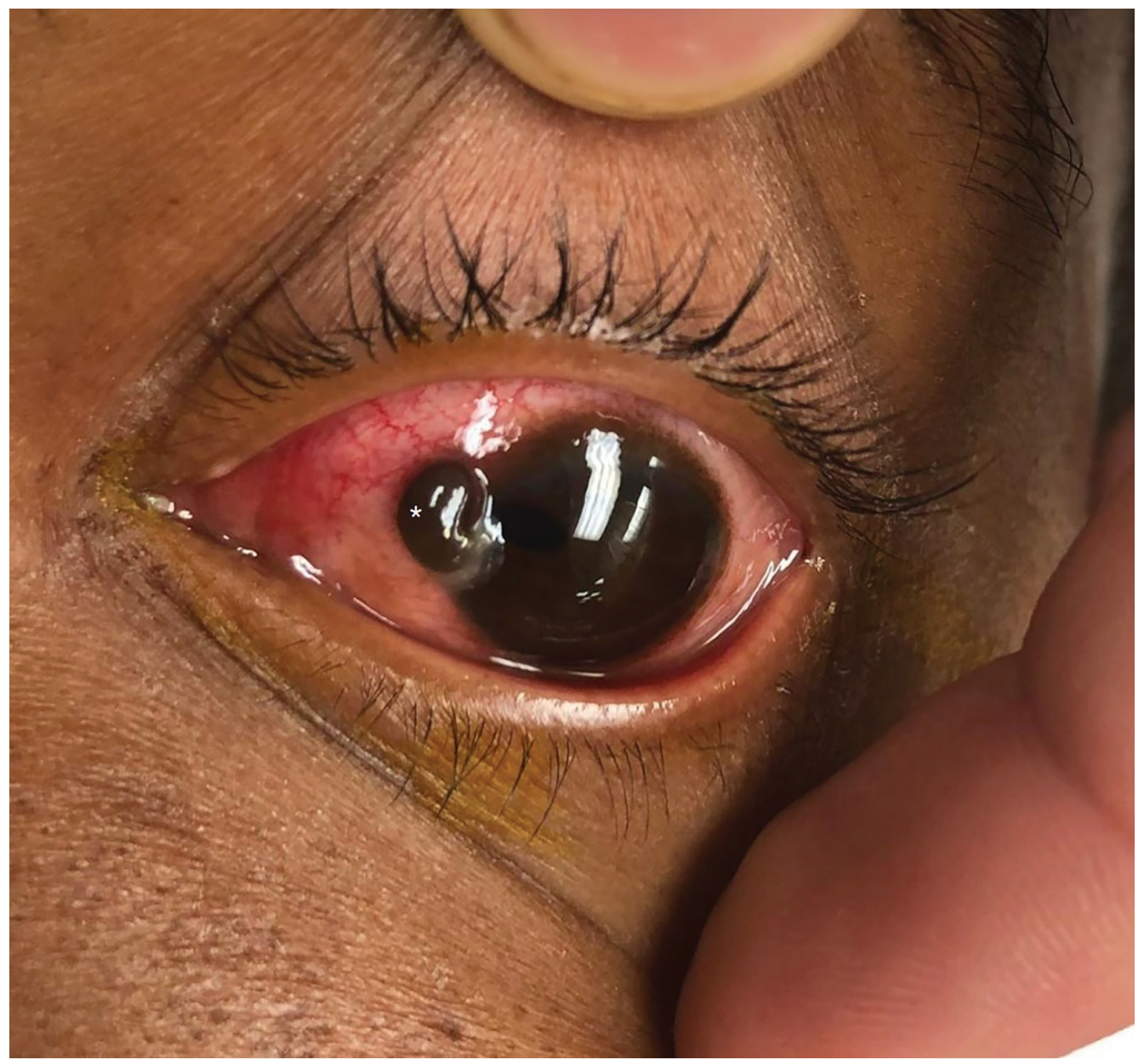

up is vital to outpatient success. There should be a low threshold to admit if suspecting compliance issues. $^{30}$

\section{Retinal Detachment}

Retinal detachment is the separation of the neurosensory retina from the underlying retinal pigmented epithelium. These 2 layers are normally opposed by 2 forces: mechanical (interdigitation of microvilli) and metabolic (fluid flowing from the vitreous to the choroid). Ocular contusion causes the globe to compress with subsequent rebound decompression in the afferent pupillary direction. These movements cause the vitreous to pull on the retina and may lead to retinal breaks. In addition, trauma may liquefy the vitreous. The combination of a retinal break and liquefied vitreous can lead to accumulation of subretinal fluid with subsequent retinal detachment (Figure 9). ${ }^{31}$

Patients may complain of new-onset flashes, floaters, and visual field defects. Examination may show decreased visual acuity, loss of a red reflex, or rAPD. Direct ophthalmoscopy may visualize a retinal detachment but has low sensitivity due to its narrow field of view. ${ }^{32}$ Use of a slit lamp can visualize brown pigmented cells in the anterior vitreous 
Figure 7. 8-ball hyphema. External photograph of the right eye with deoxygenated blood filling the anterior chamber, also known as an 8-ball hyphema.

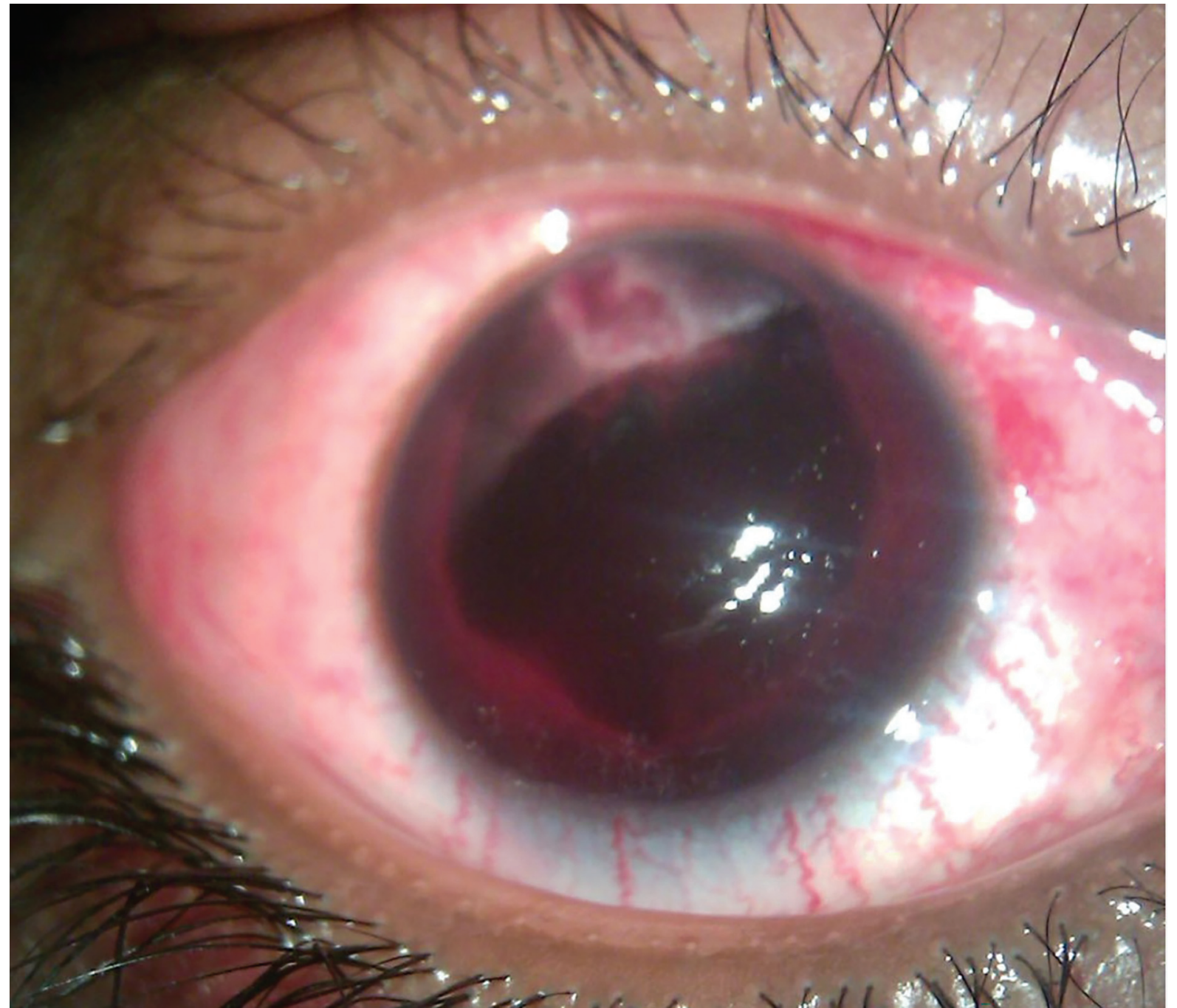

(Shafer's sign or "tobacco dust"). ${ }^{33}$ If the view of the retina is obscured, ultrasonography can assist in diagnosis with high sensitivity and specificity. ${ }^{34}$

\section{Eyelid Laceration}

The character and location of eyelid lacerations dictate management. ${ }^{35}$ In the presence of an open globe, manipulation of the lid should be deferred. $^{28,35}$ Referral to ophthalmology is appropriate for full-thickness lacerations, laceration with orbital fat prolapse, laceration involving the lid margins or lacrimal system, and lid trauma with avulsion (Figure 10). ${ }^{36}$ Suspect canalicular involvement with injuries to the medial canthus. ${ }^{37}$ Canalicular damage is common in eyelid lacerations from dog bites due to penetrating and shearing forces. ${ }^{38}$ Management of dog bites includes copious irrigation, debridement of devitalized tissue, closure of the wound, and prophylactic antibiotics. Postexposure prophylaxis for rabies and tetanus may be considered. ${ }^{39,40}$ Superficial closure can be performed with 6-0 nylon or 6-0 prolene sutures; however, absorbable sutures such as 6-0 plain gut can be used in children. ${ }^{41}$ 6-0 silk sutures can be used to reapproximate the eyelid margin, whereas deep closure should be performed using 5-0 or 6-0 vicryl sutures. ${ }^{41}$ The repair should occur within 12 hours of injury for optimal outcomes. ${ }^{35,36}$

\section{Telemedicine}

Telemedicine serves the needs of patients in remote locations without in-person access to 
Figure 8. Grade I Hyphema. External photograph of the left eye with blood covering less than $1 / 3$ of the anterior chamber thus classified as a grade I hyphema (*). Note the color of the blood compared with the deoxygenated blood in Figure 6.

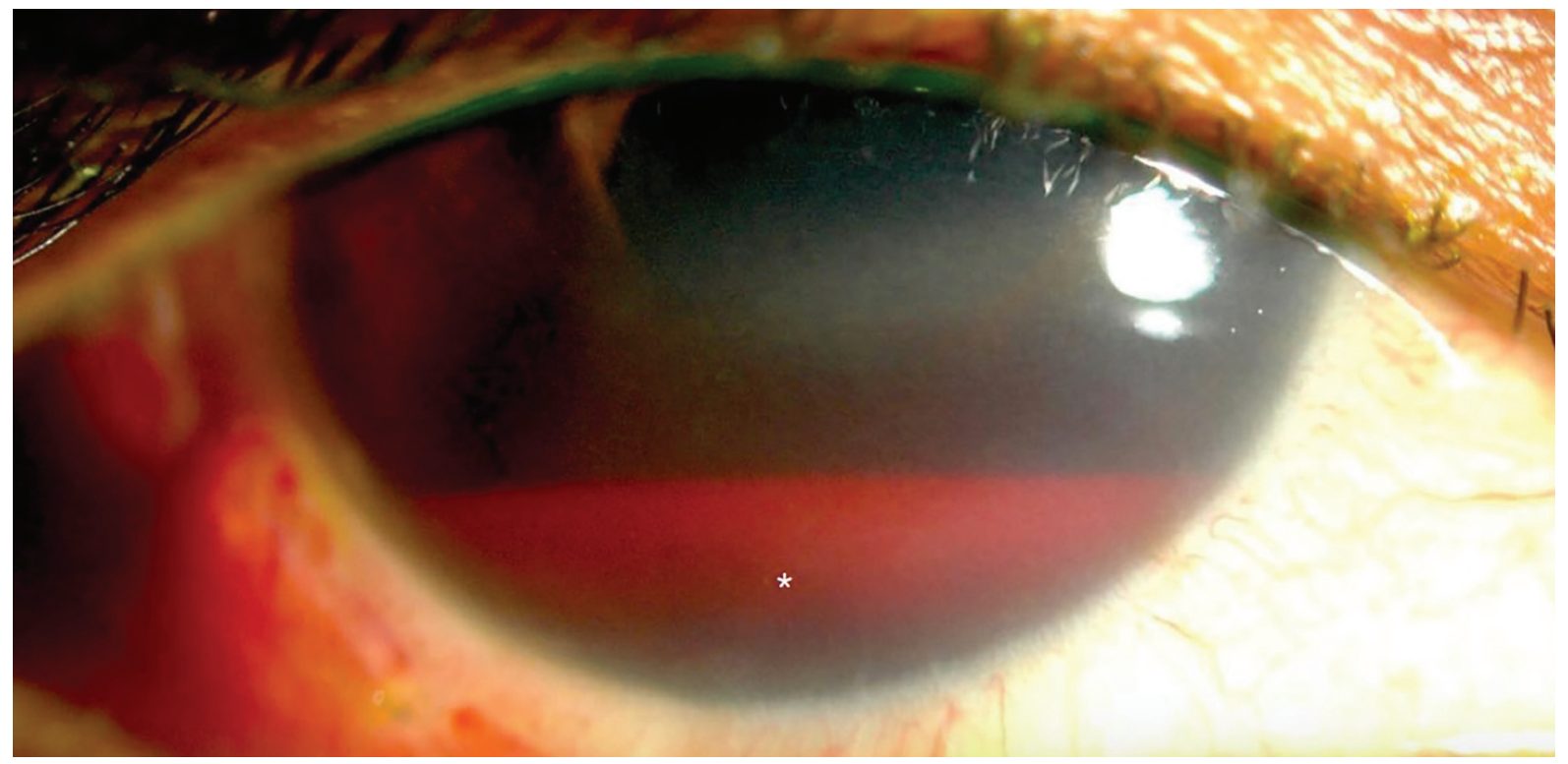

Figure 9. Retinal detachment with retinal tear. Wide-field fundus photograph of the right eye demonstrating a superotemporal horseshoe retinal tear $\left(^{*}\right)$ with retinal detachment extending into the macula. Note the demarcation line and elevated retinal vessels of the superotemporal arcade compared with the other retinal vasculature.

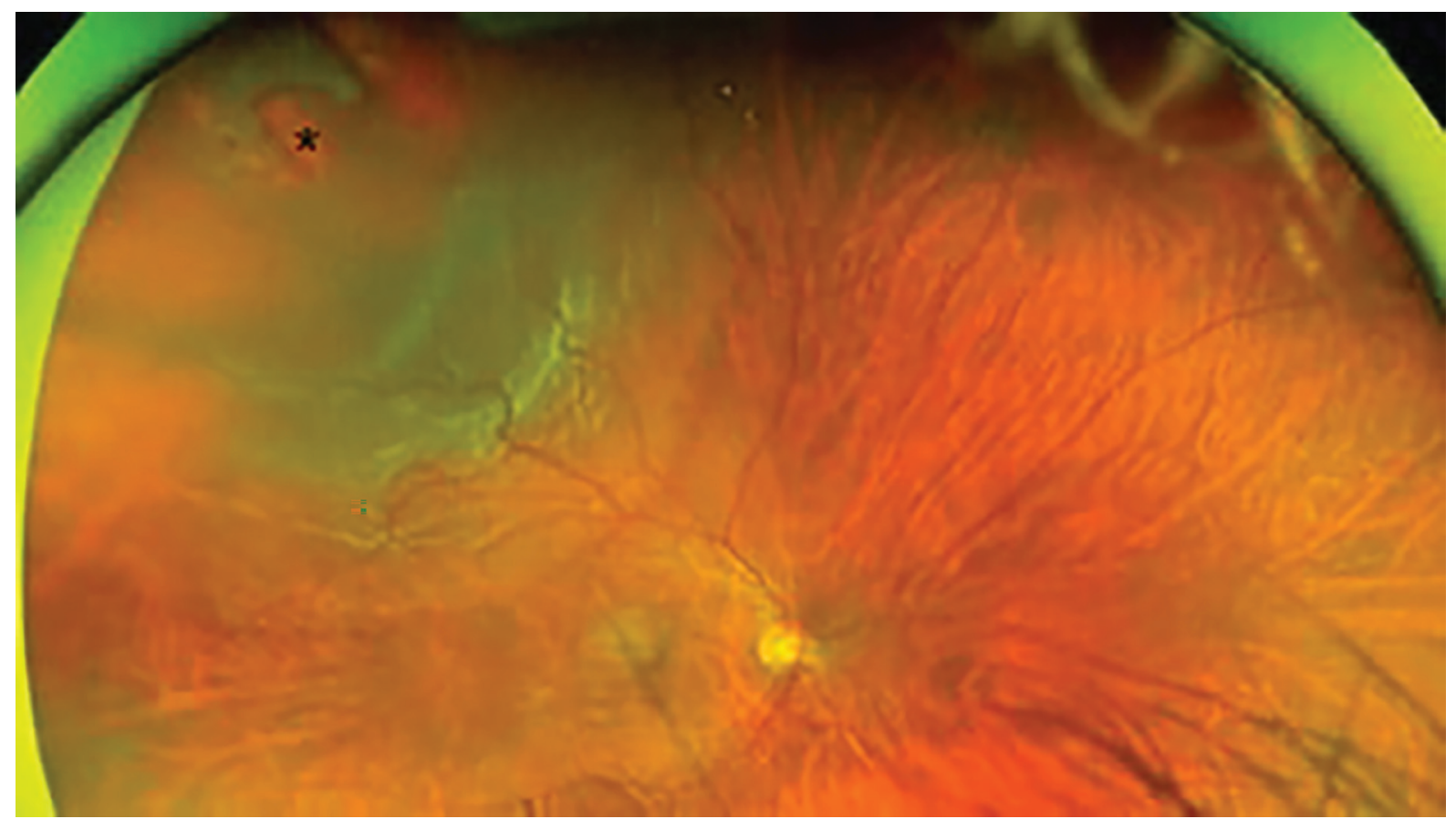


Figure 10. Marginal eyelid laceration. External photograph of the right eye showing a full-thickness marginal lid laceration with orbital fat prolapse (*) and involvement of the canalicular system. Note the exposed tarsal plate $\left({ }^{\wedge}\right)$ on the temporal portion of the laceration at the eyelid margin.

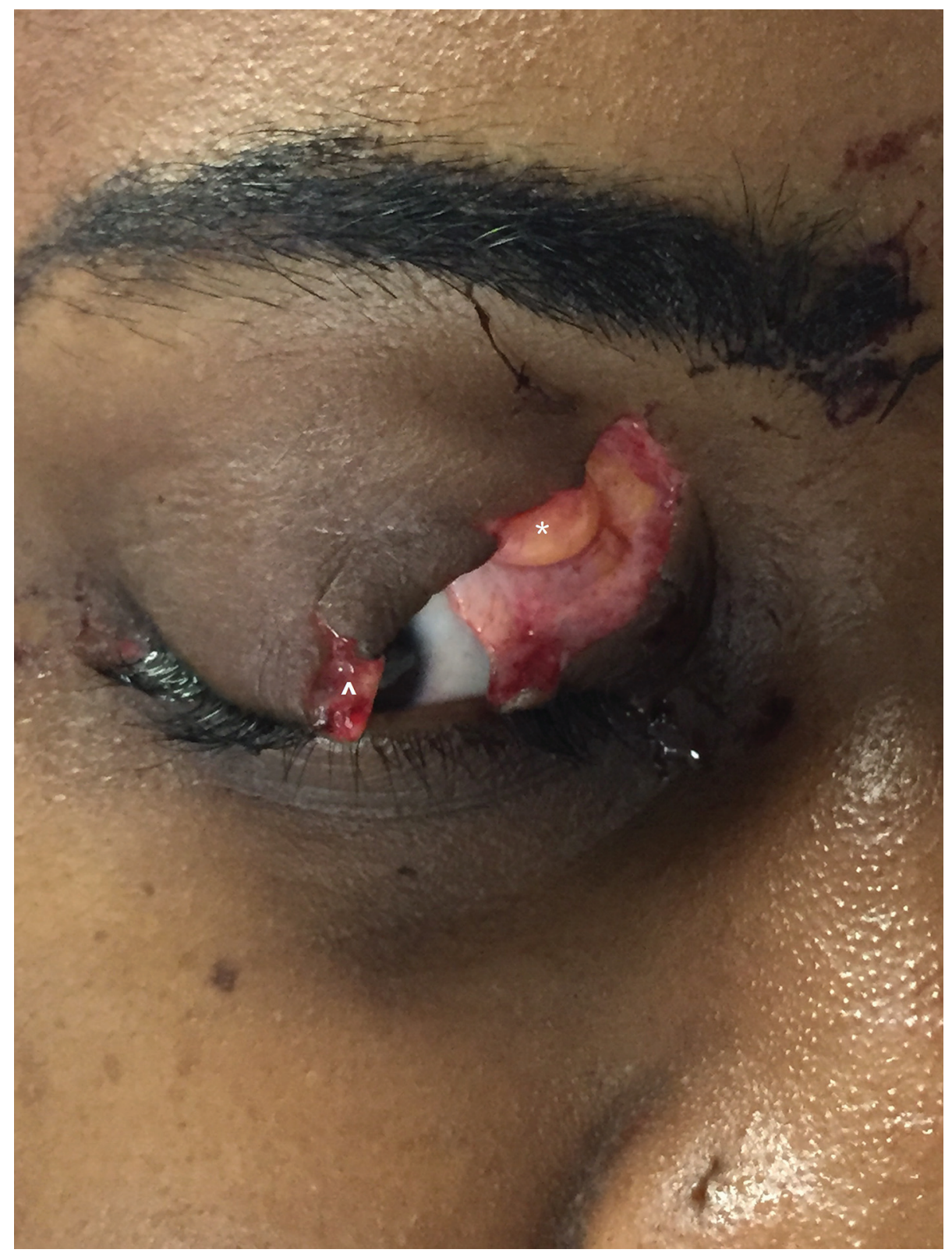

subspecialty care. It has advanced with the advent of HIPAA-compliant telecommunication applications. Teleophthalmology serves as a clinical screening tool for age-related macular degeneration, retinopathy of prematurity, and diabetic retinopathy. ${ }^{42,43}$ Teleophthalmology is also applicable to an emergent setting when a remote location lacks access to an in-person ophthalmologist. ${ }^{42}$ Live audiovisual services connecting remote ophthalmologists with frontline health care providers can assist in making precise and accurate triage decisions in patients with ocular injuries.

\section{Conclusion}

Non-ophthalmology health care providers may initially manage eye trauma, and adopting a methodological approach reduces the chance of missing injuries. Chemical eye injuries require immediate and copious irrigation. Clinicians should document 
a general history, ocular history, external examination, and internal examination. Primary care providers should recognize the key history, physical examination, pathophysiology, and basic management of common, serious eye injuries that require prompt ophthalmology referral.

To see this article online, please go to: http://jabfm.org/content/ 34/5/1018.full.

\section{References}

1. Vaziri K, Schwartz SG, Flynn HW, Jr., Kishor KS, Moshfeghi AA. Eye-related emergency department visits in the United States, 2010. Ophthalmology 2016;123:917-9.

2. Channa R, Zafar SN, Canner JK, Haring RS, Schneider EB, Friedman DS. Epidemiology of eyerelated emergency department visits. JAMA Ophthalmol 2016;134:312-9.

3. Physician Shortage Spikes Demand in Several Specialties. NE7M CareerCenter.

4. Conrad DR. Ocular Trauma: Principles and Practice. Ferenc Kuhn, Dante J. Pieramici (Eds.). Thieme (2002). Canadian J Ophthalmol 2004;39:802.

5. Harlan JB, Jr., Pieramici DJ. Evaluation of patients with ocular trauma. Ophthalmol Clin North Am 2002;15:153-61.

6. Blice JP. Ocular injuries, triage, and management in maxillofacial trauma. Atlas Oral Maxillofac Surg Clin North Am 2013;21:97-103.

7. Bord SP, Linden J. Trauma to the globe and orbit. Emerg Med Clin North Am 2008;26:97-123.

8. Janda AM. Ocular trauma: triage and treatment. Postgrad Med 1991;90:51-2, 55-60. Article. 01/01/.

9. Machiele R, Motlagh M, Patel BC. Intraocular Pressure. StatPearls. 2020.

10. Dharmasena A, Park DY, Vishwanath M. Does high intraocular pressure exclude an open globe injury? Int J Ophthalmol 2014;7:389-90.

11. Lima V, Burt B, Leibovitch I, Prabhakaran V, Goldberg RA, Selva D. Orbital compartment syndrome: the ophthalmic surgical emergency. Surv Ophthalmol 2009;54:441-9.

12. Belliveau AP, Somani AN, Dossani RH. Pupillary Light Reflex. StatPearls. StatPearls Publishing; 2020.

13. Lin KY, Ngai P, Echegoyen JC, Tao JP. Imaging in orbital trauma. Saudi J Ophthalmol 2012;26: 427-32.

14. Kubal WS. Imaging of orbital trauma. RadioGraphics 2008;28:1729-39.

15. Singh P, Tyagi M, Kumar Y, Gupta KK, Sharma PD. Ocular chemical injuries and their management. Oman J Ophthalmol 2013;6:83-6.

16. Bates A, Zanaboni A. Ocular Burns. StatPearls. StatPearls Publishing; 2020.
17. Kuckelkorn R, Schrage N, Keller G, Redbrake C. Emergency treatment of chemical and thermal eye burns. Acta Ophthalmol Scand 2002;80:4-10. 2002/ 02/01.

18. Eslani M, Baradaran-Rafii A, Movahedan A, Djalilian AR. The ocular surface chemical burns. J Ophthalmol 2014;2014:196827.

19. Koenen L, Waseem M. Orbital Floor (Blowout) Fracture. StatPearls. StatPearls Publishing; 2020.

20. Waterhouse N, Lyne J, Urdang M, Garey L. An investigation into the mechanism of orbital blowout fractures. Br J Plast Surg 1999;52:607-12.

21. Thiagarajah C, Kersten RC. Medial wall fracture: an update. Craniomaxillofac Trauma Reconstr 2009;2:135-39.

22. Joseph JM, Glavas IP. Orbital fractures: a review. Clin Ophthalmol 2011;5:95-100.

23. Duane TD, Schatz NJ, Caputo AR. Pseudo-Duane's retraction syndrome. Trans Am Ophthalmol Soc 1976;74:122-32.

24. Zapala J, Bartkowski AM, Bartkowski SB. Lacrimal drainage system obstruction: management and results obtained in 70 patients. J Craniomaxillofac Surg 1992;20:178-83.

25. Burnstine MA. Clinical recommendations for repair of isolated orbital floor fractures: an evidence-based analysis. Ophthalmology 2002;109:1207-10.

26. Roth FS, Koshy JC, Goldberg JS, Soparkar CNS. Pearls of orbital trauma management. Semin Plast Surg 2010;24:398-410.

27. Mishra A, Baranwal VK, Parihar JKS, Verma AK. Simple laceration wound of the eyelids? Always remember to look under the lids!. Med J Armed Forces India 2013;69:301-4.

28. Blair K, Alhadi SA, Czyz CN. Globe Rupture. StatPearls. 2020.

29. Wilson FM. Traumatic hyphema. Pathogenesis and management. Ophthalmology 1980;87:910-9.

30. Walton $W$, Von Hagen S, Grigorian R, Zarbin $M$. Management of traumatic hyphema. Surv Ophthalmol 2002;47:297-334.

31. Ghazi NG, Green WR. Pathology and pathogenesis of retinal detachment. Eye (Lond) 2002; 16:411-21.

32. Kang HK, Luff AJ. Management of retinal detachment: a guide for non-ophthalmologists. BMJ 2008;336:1235-40.

33. Tanner V, Harle D, Tan J, Foote B, Williamson TH, Chignell AH. Acute posterior vitreous detachment: the predictive value of vitreous pigment and symptomatology. $\mathrm{Br} \mathrm{J}$ Ophthalmol 2000;84:1264-68.

34. Lahham S, Shniter I, Thompson M, et al. Point-ofcare ultrasonography in the diagnosis of retinal detachment, vitreous hemorrhage, and vitreous detachment in the emergency department. JAMA Netw Open 2019;2:e192162-e192162. 
35. Cochran ML, Czyz CN. Eyelid Laceration. StatPearls. StatPearls Publishing; 2020.

36. Brown DJ, Jaffe JE, Henson JK. Advanced laceration management. Emerg Med Clin North Am 2007;25:83-99.

37. Murchison AP, Bilyk JR. A Practical Approach to Canalicular Lacerations. Available from: https:// www.reviewofophthalmology.com/article/a-practicalapproach-to-canalicular-lacerations. Published May $10,2017$.

38. Savar A, Kirszrot J, Rubin PAD. Canalicular involvement in $\operatorname{dog}$ bite related eyelid lacerations. Ophthalmic Plast Reconstr Surg 2008;24:296-98.

39. Nagendran S, Litwin AS, Sira M, Norris J, Malhotra R, Dheansa B. Management of facial and periocular dog bites: a review of 104 cases. Eur J Plast Surg 2014;37:595-98.

40. Stevens DL, Bisno AL, Chambers HF, et al. Practice guidelines for the diagnosis and management of skin and soft tissue infections: 2014 update by the Infectious Diseases Society of America. Clin Inf Dis 2014;59:e10-e52.

41. Chang EL, Rubin PA. Management of complex eyelid lacerations. Int Ophthalmol Clin 2002;42:187201.

42. Rathi S, Tsui E, Mehta N, Zahid S, Schuman JS. The current state of teleophthalmology in the United States. Ophthalmology 2017;124:1729-34. Dec.

43. Li HK. Telemedicine and ophthalmology. Surv Ophthalmol 1999;44:61-72. 